\title{
ON THE MINIMAL NUMBER OF CRITICAL POINTS OF SMOOTH MAPS BETWEEN CLOSED MANIFOLDS
}

\author{
DORIN ANDRICA, LOUIS FUNAR, AND ELENA KUDRYAVTSEVA
}

\section{IntRoduCtion AND STATEMENTS}

If $M, N$ are connected manifolds, possibly with boundary, consider smooth maps $f: M \rightarrow N$ with $\partial M=f^{-1}(\partial N)$ (i.e. proper) such that $f$ has no critical points on $\partial M$. Let $\varphi\left(M^{m}, N^{k}\right)$ denote the minimal number of critical points of such smooth proper maps between the manifolds $M^{m}$ and $N^{k}$ of dimensions $m$ and respectively $k$. In this paper we will consider only the case when $m \geq k \geq 2$, unless the opposite is explicitly stated. The main problem in this area is to characterize those pairs of manifolds for which $\varphi$ is finite non-zero and then to compute its value (see [7], p.617).

Let $V=f^{-1}(f(x))$, where $x$ is a critical point. Following King (see [20]) the singular point $x$ is called cone-like if it admits a cone neighborhood in $V$, i.e. there exists some closed manifold $L \subset V \backslash\{x\}$ and a neighborhood $N$ of $x$ in $V$ which is homeomorphic to the cone $C(L)$ over $L$. Recall that the cone is defined as the quotient $C(L)=L \times(0,1] / L \times\{1\}$. Then the manifold $L$ is called the local link at $x$. If $x$ is not cone-like then $x$ (and also $V$ ) are called wild.

A well-known theorem of Łojasiewicz (see e.g. [22]) states that real analytic maps have cone-like singularities. However smooth functions might have wild singularities. The first examples of smooth maps with isolated wild singularities were obtained by Takens (see [29]) in codimension 3.

The main result obtained in [1] is the following characterization of $\varphi$ in small codimension:

Theorem 1.1. Consider two closed connected manifolds with finite $\varphi\left(M^{m}, N^{k}\right)$ and $k \geq 2$.

(1) If $0 \leq m-k \leq 2$ then $\varphi\left(M^{m}, N^{k}\right) \in\{0,1\}$, except for the exceptional pairs of dimensions $(m, k) \in\{(2,2),(4,3),(4,2)\}$.

(2) If $m-k=3$ assume moreover that there exists a smooth map $f: M \rightarrow N$ with finitely many critical points, all being cone-like. Then $\varphi\left(M^{m}, N^{k}\right) \in\{0,1\}$, except for the additional set of exceptional pairs of dimensions $(m, k) \in\{(5,2),(6,3),(8,5)\}$.

Moreover, under the finiteness hypothesis, $\varphi(M, N)=1$ if and only if $M$ is the connected sum of a smooth fibration over $N$ with an exotic sphere and not a fibration itself.

Remark 1.1. The proof provided in [1], where the result is stated for all singularities of codimension at most three, actually works only for smooth maps with cone-like singularities. As explained in the addendum [2] (and using [7,9]) isolated singularities of smooth functions in codimension at most 2 with $k \geq 2$ are cone-like. Thus the proof is complete for codimension at most 2. In [16] we will analyse wild codimension three singularities.

1991 Mathematics Subject Classification. $57 \mathrm{R} \mathrm{45,58} \mathrm{K} \mathrm{05,} 57$ R 60, 14 P 25, 57 R 70.

Key words and phrases. Critical point, smooth map, ramified covering.

The first author was partially supported by the CNCSIS Grant No.130/2006,CEEX III/P-INT-VIZ and CNCSIS Grant No.1467/2007. The second author was partially supported by the ANR RepSurf: ANR-06-BLAN-0311. The third author was partially supported by the grants RFBR (projects 07-01-00648, 05-01-22002 NTsNI), RF President Programme "Support of leading scientific schools" (grant SS-660.2008.1) and the grant of National Scientific Projects 2.1.1.7988. 
There are two essential ingredients in this result. First, there are local obstructions to the existence of isolated singularities, namely the germs of smooth maps $\mathbb{R}^{m} \rightarrow \mathbb{R}^{k}$ having an isolated singularity at origin are actually locally topologically equivalent to a projection. Thus, these maps are topological fibrations. Second, singular points located in a disk cluster together.

The behavior of $\varphi$ in the exceptional dimensions is rather different. For instance, there are topological obstructions preventing singular points to cluster together. Specifically, the authors of [15] proved the following:

Theorem 1.2. If $n \in\{2,4,8\}$ then

$$
\varphi\left(\# e S^{n} \times S^{n}, S^{n+1}\right)=2 e+2 .
$$

In particular $\varphi$ can take arbitrary large (even) values in the exceptional dimensions $(4,3)$ and $(8,5)$.

Very little is known for the other exceptional and generic (i.e. $m-k \geq 4$ ) cases and even the case of pairs of spheres is not completely settled yet. We proved in [1] the following partial results for maps between spheres:

Theorem 1.3. (1) The values of $m>k \geq 1$ for which $\varphi\left(S^{m}, S^{k}\right)=0$ are exactly those arising in the Hopf fibrations i.e. $k \in\{2,4,8\}$ and $m=2 k-1$.

(2) One has $\varphi\left(S^{4}, S^{3}\right)=\varphi\left(S^{8}, S^{5}\right)=\varphi\left(S^{16}, S^{9}\right)=2$.

(3) If $1<k<m \leq 2 k-3$ then $\varphi\left(S^{m}, S^{k}\right)=\infty$.

(4) If $\varphi\left(S^{2 k-2}, S^{k}\right)$ is finite and $k \geq 2$ then $k \in\{2,3,5,9\}$.

Remark 1.2. It is not presently known which situation is more common, in general: $\varphi$ is bounded in terms only of the dimensions (as in small codimension non-exceptional cases) or else $\varphi$ is unbounded, as in the exceptional dimensions $(4,3)$ and $(8,5)$.

A particularly interesting case is the pair of dimensions $(4,2)$. The existence of Lefschetz fibrations provides many examples of closed 4-manifolds $M^{4}$ which have finite $\varphi\left(M^{4}, S^{2}\right)$.

Let $M^{4}$ be a closed, connected oriented and smooth 4-manifold and $\Sigma$ be a closed connected oriented surface. A Lefschetz fibration is a smooth map $f: M \rightarrow \Sigma$ such that $f$ is injective on the set of critical points and such that near each critical point $f$ is locally of the form $f\left(z_{1}, z_{2}\right)=z_{1}^{2}+z_{2}^{2}$, in local complex coordinates which are compatible with the orientations of $M$ and $\Sigma$.

Lefschetz fibrations are complex analogues of Morse functions. Every symplectic manifold admits a Lefschetz pencil (see [10]) which induces a Lefschetz fibration of the manifold obtained by blowing up finitely many points (see also [11] for a survey). Conversely, Gompf showed that any 4-manifold with a Lefschetz pencil admits a symplectic structure, provided that the fibers are non-trivial in homology (see [17]). Thus $\varphi$ can be estimated from above by means of its analogue for Lefschetz fibrations between symplectic manifolds.

There is a more general notion, namely that of an achiral Lefschetz fibration where one drops the assumption that the local complex coordinates define compatible orientations. Harer proved in [18] that a 4-dimensional manifold having a handlebody decomposition with handles of index less than or equal to two admits an achiral Lefschetz fibration over the disk with bounded fibers. Moreover, Etnyre and Fuller ([13]) showed that for any smooth, closed, simply-connected oriented 4-manifold $M$ the connected sum $M \# S^{2} \times S^{2}$ admits an achiral Lefschetz fibration over $S^{2}$. It is still not known whether all smooth, closed simply connected oriented 4-manifolds $M$ admit achiral Lefschetz fibrations and this problem is related to the existence of handlebody decompositions without index one handles on such manifolds. We believe that the simply connected closed oriented 4-manifolds $M$ with finite $\varphi\left(M, S^{2}\right)$ are precisely those admitting achiral Lefschetz fibrations. There are only a few examples of manifolds not admitting achiral Lefschetz fibrations, for instance $\#_{n} S^{1} \times S^{3}$, for $n \geq 2$ (see [17]). 
The number of critical fibers of a Lefschetz fibration of a four-manifold $M^{4}$ and the genera of the fiber and the base determine the Euler characteristic $\chi\left(M^{4}\right)$. A conjecture of Gompf claims that a symplectic four-manifold with $b_{+}>1$ will have non-negative Euler characteristic and in particular if true, it would follow that Lefschetz fibrations on such manifolds over $S^{2}$ have at least $4 g-4$ singular fibers, where $g$ denotes the fiber genus.

Stipsicz provided in $[27,28]$ lower bounds for the numbers of singular fibers in Lefschetz fibrations:

Theorem 1.4. A nontrivial genus $g$ Lefschetz fibration over $S^{2}$ has at least $\frac{1}{5}(8 g-4)$ irreducible critical fibers. Moreover, if the 4-manifold has $b_{+}=1, g \geq 6$ is even then we have at least $2 g+4$ critical fibers, while for odd $g \geq 15$ we have at least $2 g+10$ critical fibers.

Korkmaz and Ozbagci considered the minimal number $N(g, h)$ of singular fibers in a Lefschetz fibration with at least one singular fiber whose generic fiber is supposed to be connected and the fibration is relatively minimal, i.e. no fiber contains a $(-1)$ sphere that is an embedded sphere of self-intersection -1 , where $g$ is the genus of the fiber and $h$ the genus of the base. They proved in [21] a result which parallels theorem 1.1 of [1], namely:

Theorem 1.5. We have $N(g, h)=1$ if and only if $g \geq 3$ and $h \geq 2$. Moreover, $N(g, 1)>1$ for all $g \geq 1$.

Other inequalities which give control on the number of critical points that can cluster in a fiber are proved by Braungardt and Kotschick in [6].

Remark 1.3. Using a construction of Matsumoto of singular fibrations by tori (see [23]) one can prove that $\varphi\left(S^{4}, S^{2}\right)=1$. It is easy to see that $\varphi\left(S^{4}, S^{2}\right) \leq 2$ by considering the composition of the maps $S^{4} \rightarrow S^{3}$ and $S^{3} \rightarrow S^{2}$, where the second one is the Hopf fibration and the former one is the suspension of the Hopf fibration. This construction yields two smooth maps, each one with two singularities which lie either in two distinct fibers or in the same fiber, depending on the position of the suspension points with respect to the fibers of the Hopf fibration.

In the present paper we add new information by computing $\varphi(M, N)$ for all closed connected surfaces and thus completing the results from [1] with the non-orientable case.

Observe that a smooth map $f: Y \rightarrow X$ between surfaces has finitely many critical points if and only if it is a ramified covering. Furthermore, $\varphi(Y, X)$ is the minimal number of ramification points of a covering $Y \rightarrow X$.

Denote by $\lceil r\rceil$ the smallest integer greater than or equal to $r$ and by $\lfloor x\rfloor$ the largest number smaller or equal to $x$. Write, when $\chi(N)<0$ and $\chi(M) \leq 0$ :

$$
|\chi(M)|=d|\chi(N)|+v, \text { where } d, v \in \mathbb{Z}_{+}, 0 \leq v<|\chi(N)|,
$$

so that

$$
d=\left\lfloor\frac{\chi(M)}{\chi(N)}\right\rfloor .
$$

When $N$ is a non-orientable surface let $\widehat{N}$ denote the orientable double cover of $N$.

Theorem 1.6. Let $M$ and $N$ be connected closed surfaces.

I. Assume that $M$ and $N$ are orientable.

(a) If $\chi(M)>\chi(N)$ then $\varphi(M, N)=\infty$.

(b) If $M \neq S^{2}$ then $\varphi\left(M, S^{2}\right)=3$ and $\varphi\left(S^{2}, S^{2}\right)=0$.

(c) If $N$ is the torus $S^{1} \times S^{1}$ then

$$
\varphi\left(M, S^{1} \times S^{1}\right)= \begin{cases}1, & \text { if } \chi(M)<0 \\ 0, & \text { if } M=S^{1} \times S^{1} \\ \infty, & \text { if } M=S^{2} .\end{cases}
$$


(d) If $\chi(N)<0$, then

$$
\varphi(M, N)=\left\{\begin{array}{cl}
\left\lceil\frac{v}{d-1}\right\rceil, & \text { if } d \geq 2 \\
0, & \text { if } M=N \\
\infty, & \text { otherwise }
\end{array}\right.
$$

II. Suppose that $M$ and $N$ are non-orientable.

(a) If $N=\mathbb{R P}^{2}$ then

$$
\varphi\left(M, \mathbb{R P}^{2}\right)= \begin{cases}0, & \text { if } M=\mathbb{R P}^{2} \\ 2, & \text { otherwise. }\end{cases}
$$

(b) When $N$ is the Klein bottle we have

$$
\varphi\left(M, \mathbb{R P}^{2} \# \mathbb{R P}^{2}\right)=\left\{\begin{array}{cl}
1, & \text { if } \chi(M) \equiv 0(\bmod 2) \\
0, & \text { if } M=\mathbb{R P}^{2} \# \mathbb{R P}^{2} ; \\
\infty, & \text { if } \chi(M) \neq \equiv(\bmod 2)
\end{array}\right.
$$

(c) Assume from now on that $\chi(N)<0$, so $N$ is not $\mathbb{R P}^{2}$ nor the Klein bottle.

(i) If $\chi(M) \geq 2 \chi(N)$ then

$$
\varphi(M, N)= \begin{cases}0, & \text { if } \chi(M)=2 \chi(N) \\ 0, & \text { if } M=N \\ \infty, & \text { otherwise }\end{cases}
$$

(ii) If $\chi(M)<2 \chi(N)$.

(A) Assume that $\chi(N) \equiv 0(\bmod 2)$. Then

$$
\varphi(M, N)=\left\{\begin{array}{cl}
\left\lceil\frac{v}{d-1}\right\rceil, & \text { if } \chi(M) \equiv \chi(N) \equiv 0(\bmod 2) \\
\infty, & \text { if } \chi(M) \equiv 1(\bmod 2), \chi(N) \equiv 0(\bmod 2) .
\end{array}\right.
$$

(B) Suppose that $\chi(N) \equiv 1(\bmod 2)$. Then

$$
\varphi(M, N)= \begin{cases}\left\lceil\frac{v}{d-1}\right\rceil, & \text { if } d \equiv \chi(M)(\bmod 2) \\ \left\lceil\frac{v+|\chi(N)|}{d-2}\right\rceil, & \text { if } d \not \equiv \chi(M)(\bmod 2), d \geq 3 \\ \infty, & \text { if } d \neq \equiv \chi(M)(\bmod 2), d=2\end{cases}
$$

III. Suppose that $M$ is non-orientable and $N$ is orientable. Then $\varphi(M, N)=\infty$. IV. $M$ is orientable and $N$ is not orientable.

(a) If $\chi(N)<0$

$$
\varphi(M, N)=\left\{\begin{array}{cl}
\left\lceil\frac{v+|\chi(N)|}{d-1}\right\rceil, & \text { if } d \text { is odd } d \geq 5 \\
\left\lceil\frac{2 v}{d-2}\right\rceil, & \text { if } d \text { is even } d \geq 4 \\
0, & \text { if } M=\widehat{N} \\
\infty, & \text { if } M \neq \widehat{N}, d \leq 3
\end{array}\right.
$$

where $\widehat{N}$ denotes the orientable double cover of $N$.

(b) If $N=\mathbb{R P}^{2}$ then

(c) If $N=\mathbb{R} \mathbb{P}^{2} \# \mathbb{R P}^{2}$ then

$$
\varphi\left(M, \mathbb{R P}^{2}\right)= \begin{cases}3, & \text { if } \chi(M) \leq 0 \\ 0, & \text { if } M=S^{2} .\end{cases}
$$

$$
\varphi\left(M, \mathbb{R} \mathbb{P}^{2} \# \mathbb{R P}^{2}\right)= \begin{cases}1, & \text { if } \chi(M)<0 \\ 0, & \text { if } M=S^{1} \times S^{1} \\ \infty, & \text { if } M=S^{2}\end{cases}
$$


Remark 1.4. Computations were previously done for orientable surfaces in [1] and in [24] it was proved that $\varphi(Y, X)$ is infinite when $\chi(Y)>\chi(X)$.

Moreover, recent results of Bogatyi, Gonçalves, Kudryavtseva and Zieschang ([4, 5]) show that the minimal number of critical points can be achieved by using maps $f: Y \rightarrow X$ which are primitive branched coverings, i.e. maps inducing surjective mappings at the level of fundamental groups.

\section{Proof of THeOREM 1.6}

2.1. The existence of branched coverings with prescribed ramification. The proof is based upon results of Edmonds, Kulkarni and Stong ([12]) that gave necessary and sufficient conditions for the existence of a covering of a surface with prescribed degree and branch data (i.e. collection of ramification orders over each branch point). We have from [12], Proposition 2.8:

Proposition 2.1. Let $M, N$ be connected closed surfaces and $\delta \geq 2$. Suppose that $M$ is orientable if $N$ is orientable. Moreover, if $N$ is non-orientable and $\delta$ is odd or $\delta=2$ then $M$ is non-orientable. Then there is some degree $\delta$ branched covering $f: M \rightarrow N$ if and only if $\chi(M) \leq \delta \chi(N)$ and $\nu=\delta \chi(N)-\chi(M)$ is even.

Consider $M, N$ as in the hypothesis of the proposition. Assume that we have a branched covering $M \rightarrow N$ of degree $\delta$ with $r$ ramification points of ramification degrees $d_{i}, i=1, r$. Then $2 \leq d_{i} \leq \delta$ and Hurwitz' formula reads

$$
\chi(M)=\delta \chi(N)-\sum_{i=1}^{r}\left(d_{i}-1\right) .
$$

It follows from $[19,14,3,12]$ that, for any collection $2 \leq d_{i} \leq \delta, i=1, \ldots, r$, satisfying (11), the following statement holds. If $N \neq S^{2}$ and there exists a degree $\delta$ branched covering $f$ as in proposition 2.1, where either $N$ is orientable or $M$ is non-orientable, then there exists such $f$ with $r$ ramification points of ramification degrees $d_{i}, i=1, \ldots, r$.

Therefore $\varphi(M, N)$ is the minimal possible $r$ for which there exists a natural solution $\delta, d_{i}$ of the linear equation above such that $\chi(M)-\delta \chi(N)$ is even and $2 \leq d_{i} \leq \delta$ (possibly with additional assumptions related to the orientability, or $N \neq S^{2}$ ). In the case $N=S^{2}$ we only showed the necessity of the conditions (11) for the existence of the branched covering. As we will show below (see 2.5 and 2.7), such a covering exists if $N=S^{2}, r=3$ and $\delta=3-\chi(M)$.

Observe that the condition $\delta \geq 2$ is necessary, since a degree one branched covering is a homeomorphism.

We have the bounds

$$
r \leq \sum_{i=1}^{r}\left(d_{i}-1\right) \leq r(\delta-1),
$$

which imply

$$
r \leq-\chi(M)+\delta \chi(N) \leq r(\delta-1) .
$$

Conversely, if $r$ and $\delta$ satisfy the above inequalities then we can find solutions $d_{i}$ of the equations above. Thus, if $N \neq S^{2}$ and either $N$ is orientable or $M$ is non-orientable, then we need to find the smallest $r$ for which there exists a natural solution $\delta \geq 2$ to this system of inequalities, such that $\chi(M)-\delta \chi(N)$ is even.

2.2. The generic case, when $\chi(M) \leq \chi(N)<0$ and $d \geq 2$. The later inequalities are then equivalent to

$$
\frac{\chi(M)-r}{\chi(N)-r} \leq \delta \leq \frac{\chi(M)+r}{\chi(N)} .
$$


Lemma 2.1. Any solution $\delta$ of (14) satisfies $\delta \leq d$.

Proof. We have

$$
\delta \leq \frac{\chi(M)+r}{\chi(N)}=d+\frac{v-r}{|\chi(N)|}<d+1
$$

since $v<|\chi(N)|$.

If we drop the parity condition we are looking for the smallest natural number $r$ satisfying

$$
\left\lceil\frac{\chi(M)-r}{\chi(N)-r}\right\rceil \leq \frac{\chi(M)+r}{\chi(N)}
$$

Lemma 2.2. The smallest value of $r$ for which (15) is verified is given by $a=\left\lceil\frac{v}{d-1}\right\rceil$.

Proof. In fact $r=a$ verifies the inequalities because

$$
\left\lceil\frac{\chi(M)-a}{\chi(N)-a}\right\rceil=d+\left\lceil\frac{v-(d-1) a}{a-\chi(N)}\right\rceil \leq d \leq d+\frac{v-a}{|\chi(N)|}=\frac{\chi(M)+a}{\chi(N)} .
$$

On the other hand, if $r<a$ then

$$
\left\lceil\frac{\chi(M)-r}{\chi(N)-r}\right\rceil=d+\left\lceil\frac{v-(d-1) r}{r-\chi(N)}\right\rceil>d
$$

and thus

$$
\left\lceil\frac{\chi(M)-r}{\chi(N)-r}\right\rceil \geq d+1
$$

Since the left hand side is an integer, while we observed in the proof of the previous lemma that

$$
\frac{\chi(M)+r}{\chi(N)}<d+1
$$

thus the inequality (15) cannot be satisfied for any $r<a$.

Consider now the parity condition

$$
\chi(M)-\delta \chi(N) \equiv 0(\bmod 2) .
$$

A. If $\chi(N) \equiv 0(\bmod 2)$ then the parity condition is

- either satisfied for any $\delta$, namely when $\chi(M) \equiv 0(\bmod 2)$;

- or else it cannot be satisfied for any choice of $\delta$, when $\chi(M) \equiv 1(\bmod 2)$.

This proves that, in this case with $N$ orientable or $M$ non-orientable, $\varphi(M, N)$ is either $a$ or $\infty$, as claimed.

B. If $\chi(N) \equiv 1(\bmod 2)$ then the parity condition is equivalent to $\delta \equiv \chi(M)(\bmod 2)$. Moreover, recall that $\delta$ was supposed only to verify the inequalities (14). Alternatively, by lemma 2.1

$$
d+\left\lceil\frac{v-(d-1) a}{a-\chi(N)}\right\rceil \leq \delta \leq d, \quad \text { if } r=a .
$$

- If $d \equiv \chi(M)(\bmod 2)$ then $\delta=d$ verifies the parity condition and the inequalities above.

- If $d \not \equiv \chi(M)(\bmod 2)$ then

$$
-1<\frac{v-(d-1) a}{a-\chi(N)} \leq 0
$$


since otherwise $(d-2) a \geq v-\chi(N)$, thus $d \geq 3$ and $\left\lceil\frac{v}{d-1}\right\rceil=a \geq\left\lceil\frac{v-\chi(N)}{d-2}\right\rceil$ which is impossible, since $v \geq 0, \chi(N)<0$ and $d \geq 3$. In this case there is no convenient $\delta$ for $r=a$ because

$$
0 \leq \frac{v-a}{|\chi(N)|}<1, \quad\left\lceil\frac{\chi(M)-a}{\chi(N)-a}\right\rceil=d=\left\lfloor\frac{\chi(M)+a}{\chi(N)}\right\rfloor,
$$

and so the only possible value for $\delta$ would be $\delta=d$. This implies that we have to consider $r>a$ and to find then the smallest $r$ such that there exists $\delta \equiv d-1(\bmod 2)$ satisfying the inequalities:

$$
d+\left\lceil\frac{v-(d-1) r}{r-\chi(N)}\right\rceil \leq \delta \leq d+\frac{v-r}{|\chi(N)|} .
$$

This implies that the smallest $r$ with the property above has to satisfy

$$
\left\lceil\frac{v-(d-1) r}{r-\chi(N)}\right\rceil \leq-1
$$

or, equivalently, $d \geq 3$ and $r \geq\left\lceil\frac{v+|\chi(N)|}{d-2}\right]$. For the values $r=\left[\frac{v+|\chi(N)|}{d-2}\right]$ and $\delta=d-1$, which has to verify $\delta \geq 2$, both required inequalities hold. Thus, if $d=2$, we have $\varphi(M, N)=\infty$.

Summarizing it follows that, when $d \not \equiv \chi(M)(\bmod 2)$ and $N$ is orientable or $M$ is non-orientable then

$$
\varphi(M, N)= \begin{cases}\left\lceil\frac{v+|\chi(N)|}{d-2}\right\rceil, & \text { if } d \geq 3 \\ \infty, & \text { if } d=2 .\end{cases}
$$

2.3. $M, N$ are non-orientable. If $\chi(N)<0$ then the formulas above and remark 1.4 prove the claim. If $N=\mathbb{R P}^{2}$ and $M \neq \mathbb{R P}^{2}$, then the conditions (13) read

$$
r \leq \delta-\chi(M) \leq r(\delta-1)
$$

Then $r=1$ does not work since $\chi(M)<1$, while for $r=2$ the inequalities above and the parity condition have natural solutions. Thus $\varphi(M, N)=2$, if $\chi(M)<1$.

If $N=\mathbb{R P}^{2} \# \mathbb{R P}^{2}$, then the conditions (13) read

$$
r \leq-\chi(M) \leq r(\delta-1) .
$$

Thus $\chi(M) \leq-1$ and $r=1$ is convenient. However $\nu=-\chi(M)$ should be even. This implies that

$$
\varphi\left(M, \mathbb{R} \mathbb{P}^{2} \# \mathbb{R P}^{2}\right)=\left\{\begin{array}{cl}
1, & \text { if } \chi(M) \equiv 0(\bmod 2) \\
0, & \text { if } M=\mathbb{R} \mathbb{P}^{2} \# \mathbb{R P}^{2} ; \\
\infty, & \text { if } \chi(M) \neq 0(\bmod 2) .
\end{array}\right.
$$

2.4. $M$ non-orientable and $N$ orientable. It is standard that the ramified covering of an orientable manifold is orientable. Thus, in this case $\varphi(M, N)=\infty$.

2.5. $M, N$ are orientable. This was solved in [1], and it corresponds to the arguments above when $\chi(M), \chi(N)<0$ are even.

If $N=S^{2}$ and $\chi(M) \leq 0$ then the conditions (13) are

$$
r \leq 2 \delta-\chi(M) \leq r(\delta-1) .
$$

If $r \leq 2$ then $\chi(M) \geq 2$, which is impossible. For $r=3$ the inequalities above are necessary for the existence of a branched covering $M \rightarrow S^{2}$ of degree $\delta$ with $r=3$ ramification points. As we will show below, they are also sufficient for the existence of such a covering with $\delta=3-\chi(M)$ (see 2.7) and thus $\varphi\left(M, S^{2}\right)=3$, when $M \neq S^{2}$.

If $N=S^{1} \times S^{1}$ then the conditions (13) read

$$
r \leq-\chi(M) \leq r(\delta-1)
$$


and thus $r=1$ yields natural solutions for any $M$ with $\chi(M)<0$. Thus $\varphi\left(M, S^{1} \times S^{1}\right)=1$, if $M \neq S^{2}, S^{1} \times S^{1}$ and $\varphi\left(S^{2}, S^{1} \times S^{1}\right)=\infty$.

2.6. $M$ orientable and $N$ non-orientable. Assume that $\chi(M) \leq \chi(N)<0$. Then in addition to the constraints (14) we need first that the solution $\delta$ is even, since an odd degree branched covering of a non-orientable surface is also non-orientable (see e.g. [12], Proposition 2.3). Further, when $\delta=2$ then $M$ should be the orientable (non-ramified) double cover $\widehat{N}$ of $N$. This follows from the fact that the map $M \rightarrow N$ lifts to a ramified covering $M \rightarrow \widehat{N}$ (see [12], Proposition 2.7). Thus $\varphi(M, N)=\varphi(M, \widehat{N})$, which was computed in 2.5.

If $\chi(M) \leq 2 \chi(N)<0$ then write $|\chi(M)|=d^{\prime}|\chi(\widehat{N})|+v^{\prime}$, where $0 \leq v^{\prime}<|\chi(\widehat{N})|$. From 2.5 we find that

$$
\varphi(M, \widehat{N})=\left\{\begin{array}{cl}
\left\lceil\frac{v^{\prime}}{d^{\prime}-1}\right\rceil, & \text { if } d^{\prime} \geq 2 ; \\
0, & \text { if } M=\widehat{N} \\
\infty, & \text { otherwise }
\end{array}\right.
$$

Therefore, if $\chi(N)<0$

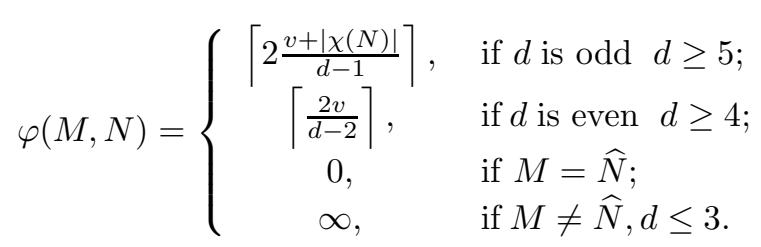

Alternatively, from $[19,14,3,12]$ for any collection $2 \leq d_{i} \leq \frac{\delta}{2}, i=1, \ldots, r$, satisfying (11), the following statement holds. If there exists a degree $\delta$ branched covering $f$ as in proposition 2.1 where $N$ is non-orientable and $M$ is orientable then there exists such $f$ with $r$ ramification points of ramification degrees $d_{i}, i=1, \ldots, r$. We can proceed as in 2.2 .

2.7. Triangulations and $N=S^{2}$. In [1] we treated separately the case $N=S^{2}$, by making use of Belyi maps (see [26]), but the proof was rather sketchy. Morris Hirsch asked us for more details and later gave us the following simple proof using triangulations. Notice that a similar construction was given by Prasolov and Sossinsky in [25], Theorem 20.6.

The algebraic topology arguments in [1] or in 2.5 show that $\varphi\left(M, S^{2}\right) \geq 3$ and it suffices to see that there exists a Belyi map having precisely 3 critical points, namely one critical point above each critical value.

There exist triangulations of the surface $M$ with any number of vertices $s \geq 1$, in particular for $s=3$. In fact, we fix the vertices and then add, inductively, a number of disjoint arcs joining the vertices such that no two arcs are homotopic, by a homotopy keeping the endpoints fixed. Consider the maximal collection of pairwise non-homotopic such arcs. Moreover the complementary regions are triangles, since otherwise we can add more arcs, contradicting the maximality. We obtained, therefore, a triangulation of $M$ having $s$ vertices, $2 s-2 \chi(M)$ triangles and $3 s-3 \chi(M)$ edges. Although each cell has its vertices among the three vertices of the triangulation, they are not necessarily distinct.

However, we need a special triangulation, namely one in which all triangles have the same (distinct) three vertices. Given $n \geq 1$ we consider the regular polygon in the hyperbolic plane (respectively the Euclidean plane, when $n=1)$ with $2(2 n+1)$ vertices and angles $\frac{2 \pi}{2 n+1}$. We identify the opposite edges by means of isometries reversing the orientation. There are then two orbits of the vertices, and around each vertex the total angle is $2 \pi$. We obtain then a closed hyperbolic surface. Let us subdivide it into equal triangles with a common vertex at the center of the polygon. This triangulation has three vertices, $2(2 n+1)$ triangles and $3(2 n+1)$ edges and thus the surface has genus $n$. Label the central vertex by 1 
and the two other vertices by 2 and 3. Then each triangle of the triangulation has the vertices 1,2 and 3 . The hyperbolic surface is oriented and thus each triangle inherits an orientation. We say that a triangle is positive if the the cyclic order of the labels of its vertices is 1,2,3 and negative otherwise. Observe that adjacent triangles have distinct signs since the order of 2, 3 is reversed.

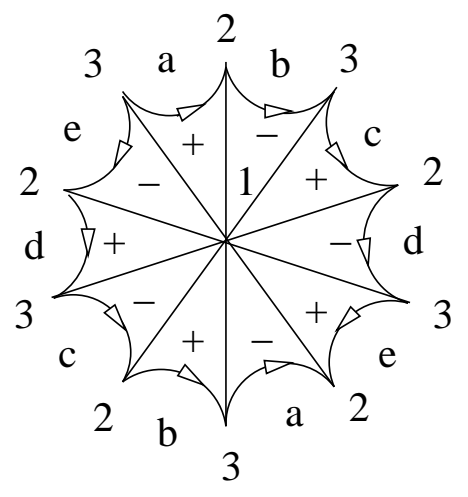

Consider next the triangulation of $S^{2}$ consisting of two triangles whose boundaries are identified. Now, map the triangulation of $M$ onto that of the sphere $S^{2}$ by mapping each triangle of $M$ to one or the other triangles of the sphere according to the sign. This yields a map $M \rightarrow S^{2}$ which is ramified only at the three vertices.

There is also a beautiful example due to John Hubbard of a Belyi function with only three critical points, obtained by considering $\Sigma \subset \mathbb{C P}^{2}$ to be the projective algebraic curve determined by the (nonhomogeneous) equation

$$
y^{2 g+1}=x^{2}-1 \text {. }
$$

The projection onto the first coordinate is a holomorphic map $M \rightarrow \mathbb{C P}^{1}$, which is ramified over $1,-1$ and $\infty$ and has only three critical points. By the Riemann-Hurwitz formula we have $\chi(M)=2-2 g$.

One can seek for the topological classification of smooth functions $f: M \rightarrow S^{2}$ with three critical points with ramification orders coiniciding with $|\operatorname{deg}(f)|$, i.e. up to the action by left multiplication by diffeomorphisms of $M$. The pull-back by $f$ of the triangulation of $S^{2}$ consisting of two triangles (with vertices at critical values) is a special triangulation of $M$, in which triangles can be given signs, according to the triangle covered on the sphere. If we label the vertices by 1,2,3 then the sign of a triangle corresponds to the cyclic order of the boundary labels. Further, if we fix a vertex, say the one labeled 1 , and look at the edges incident to it, then their endpoints are labeled only by 2 and 3 ; moreover, consecutive edges correspond to different labels, and thus the cyclic order of these labels is an alternate sequence $2,3,2, \ldots, 3$. The union of these triangles is a fundamental polygon $P$ for the surface $M$. Thus $P$ has $2(2 n+1)$ edges, where $n$ is the genus of $M$. In particular $P$ is the polygon drawn above.

Furthermore, one obtains $M$ by gluing the edges of $P$ by means of an involution $j$ on the set of edges. The gluing should satisfy the following conditions:

- the gluing reverses the orientation of the edges inherited from the circle, such that the quotient is orientable;

- $j$ preserves the labels;

- the orbit of a vertex by the permutation group generated by the involutions on the set of vertices induced by $j$ and the gluing is the set of all vertices with the same label; this means that there are precisely two vertices in the quotient $M$;

- adjacent edges are not identified by $j$. 
Thus, up to a homeomorphism of $M$ the special triangulations of $M$ correspond to polygons $P$ with an involution $j$ as above. By direct inspection it follows that there are no other involutions $j$ except the standard one from above, when the genus is at most 3.

Acknowledgements. The authors are thankful to Morris Hirsch for sharing with us his proof and the example of John Hubbard, and to Daciberg Gonçalves for suggestions and references.

\section{REFERENCES}

[1] D. Andrica and L.Funar, On smooth maps with finitely many critical points, J. London Math.Soc. 69 (2004), 783-800.

[2] D. Andrica and L. Funar, On smooth maps with finitely many critical points. Addendum, J. London Mat.Soc. 73 (2006), 231-236.

[3] I. Berstein and A.L. Edmonds, On the construction of branched coverings of low-dimensional manifolds, Trans. Amer. Math. Soc. 247 (1979), 87-124.

[4] S.A. Bogatyi, D.L. Gonçalves, E.A. Kudryavtseva and H. Zieschang, Realization of primitive branched coverings over closed surfaces, Advances in topological quantum field theory, 297-316, NATO Sci. Ser. II Math. Phys. Chem., 179, Kluwer Acad. Publ., Dordrecht, 2004.

[5] S.A. Bogatyi, D.L. Gonçalves, E.A. Kudryavtseva and H. Zieschang, Realization of primitive branched coverings over closed surfaces following the Hurwitz approach, Central Eur. J. Math. 1 (2003), 184-197.

[6] V. Braungardt and D. Kotschick, Clustering of critical points in Lefschetz fibrations and the symplectic Szpiro inequality, Trans. Amer. Math. Soc. 355 (2003), 3217-3226

[7] P.T. Church and J.G. Timourian, Differentiable maps with 0-dimensional critical set I, Pacific J. Math. 41 (1972), 615-630.

[8] P.T. Church and J.G. Timourian, Continuous maps with 0-dimensional branch set, Indiana Univ. Math. J. 23 (1974), 949-958.

[9] P.T. Church and J.G. Timourian, Differentiable maps with 0-dimensional critical set II, Indiana Univ. Math. J. 24 (1974), 17-28.

[10] S.K. Donaldson, Lefschetz pencils on symplectic manifolds, J. Differential Geom. 53 (1999), 205-236.

[11] S.K. Donaldson, Lefschetz pencils and mapping class groups, in Problems on mapping class groups and related topics, (B.Farb, Editor) 151-163, Proc. Sympos. Pure Math., 74, Amer. Math. Soc., Providence, RI, 2006.

[12] A. Edmonds, R. Kulkarni and R. Stong, Realizability of branched coverings of surfaces, Trans. Amer. Math. Soc. 282 (1984), 773-790.

[13] J.B. Etnyre and T. Fuller, Realizing 4-manifolds as achiral Lefschetz fibrations, Int. Math. Res. Not. 2006, Art. ID 70272.

[14] C.L. Ezell, Branch point structure of covering maps onto nonorientable surfaces, Trans. Amer. Math. Soc. 243 (1978), 123-133.

[15] L. Funar, C. Pintea and P. Zhang, Examples of smooth maps with finitely many critical points in dimensions $(4,3),(8,5)$ and $(16,9)$, math.GT/0803.0665.

[16] L. Funar, Smooth maps with finitely many critical points in dimensions $(4,3)$ and $(8,5)$, in preparation.

[17] R.E. Gompf and A.I. Stipsicz, 4-manifolds and Kirby calculus, Gradute Studies in Math., vol. 20, Amer. Math. Society, Providence, 1999.

[18] J.L. Harer, Pencils of curves of 4-manifolds, PhD Thesis, Univ. California, Berkeley, 1979.

[19] D.H. Husemoller, Ramified coverings of Riemann surfaces, Duke Math. J. 29 (1962), 167-174.

[20] H.C. King, Topological type of isolated singularities, Annals of Math. 107 (1978), 385-397.

[21] M. Korkmaz and B. Ozbagci, Minimal number of singular fibers in a Lefschetz fibration, Proc. Amer. Math. Soc. 129 (2001), no. 5, 1545-1549.

[22] S. Łojasiewicz, Triangulation of semi-analytic sets, Ann. Scuola Norm. Sup. Pisa (3) 18 (1964), 449-474.

[23] Y. Matsumoto, Handlebody decompositions of 4-manifolds and torus fibrations, Osaka J. Math. 33 (1996), 805-822. 
[24] C. Pintea, Continous mappings with an infinite number of topologically critical points, Annales Polonici Mathematici 67 (1997),87-93.

[25] V.V. Prasolov and B.A. Sossinsky, Knots, links, braids and 3-manifolds. An introduction to the new invariants in low-dimensional topology, Translations of Math. Monographs, 154, American Mathematical Society, 1997.

[26] G.B. Shabat and V.A. Voevodsky, Drawing curves over number fields, Grothendieck Festschrift, (P.Cartier, Ed.), Progress in Math., 88, vol. 3, Birkhauser, 1990, 199-227.

[27] A.I. Stipsicz, On the number of vanishing cycles in Lefschetz fibrations, Math. Res. Lett. 6 (1999), no. 3-4, $449-456$.

[28] A.I. Stipsicz, Singular fibers in Lefschetz fibrations on manifolds with $b_{2}^{+}=1$, Topology Appl. 117 (2002), no. $1,9-21$.

[29] F.Takens, Isolated critical points of $C^{\infty}$ and $C^{\omega}$ functions, Indag. Math. 29 (1967), 238-243.

Faculty of Mathematics and Computer Science,"Babes-Bolyai" University of Cluj, 3400 Cluj-Napoca, ROMANIA

E-mail address: dandrica@math.ubbcluj.ro

Institut Fourier BP 74, UMR 5582, Université de Grenoble I, 38402 Saint-Martin-d'Hères Cedex, France E-mail address: funar@fourier.ujf-grenoble.fr

Mathematics and Mechanics Department, Moscow State University, 119992 Moscow, Russia

E-mail address: eakudr@mech.math.msu.su 